\title{
2010 Staging System for Colon and Rectal Carcinoma
}

\author{
J. Milburn Jessup, $\mathrm{MD}^{1}$, Leonard L. Gunderson, $\mathrm{MD}^{2}$, Frederick L. Greene, $\mathrm{MD}^{3}$, Mary Kay Washington, $\mathrm{MD}^{4}$, \\ Carolyn C. Compton, MD, $\mathrm{PhD}^{5}$, Leslie H. Sobin, $\mathrm{MD}^{6}$, Bruce Minsky, $\mathrm{MD}^{7}$, Richard M. Goldberg, $\mathrm{MD}^{8}$, \\ and Stanley R. Hamilton, $\mathrm{MD}^{9}$
}

${ }^{1}$ Cancer Diagnosis Program, National Cancer Institute, Rockville, MD; ${ }^{2}$ Department of Radiation Therapy, Mayo Clinic Cancer Center, Rochester, MN; ${ }^{3}$ Department of Surgery, Carolinas Medical Center, Charlotte, NC; ${ }^{4}$ Department of Pathology, Vanderbilt University Medical Center, Nashville, TN; ${ }^{5}$ Office of Biorepositories and Biospecimen Research, National Cancer Institute, Rockville, MD; ${ }^{6}$ International Union Against Cancer, Geneva, Switzerland; ${ }^{7}$ Department of Radiation Oncology, University of Chicago Medical Center, Chicago, IL; ${ }^{8}$ Division of Hematology and Oncology, University of North Carolina at Chapel Hill, Chapel Hill, NC; ${ }^{9}$ Division of Pathology and Laboratory Medicine, UTMD Anderson Cancer Center, Houston, TX

The American Joint Committee on Cancer (AJCC) has partnered with the Annals of Surgical Oncology to publish a series of editorials that highlight revisions in staging criteria for major cancer types from the recently published 7th edition of the AJCC Cancer Staging Manual. ${ }^{1}$ Cancer registrars nationwide are now using these updates. This editorial, which focuses on the staging system for colorectal carcinoma, is the sixth in a series designed to outline the revisions of specific staging systems for the Annals of Surgical Oncology readership.

\section{THE SEVENTH EDITION STAGING SYSTEM FOR COLORECTAL CARCINOMA}

A major question facing the Hindgut Task Force of the AJCC for the 6th edition of the TNM staging system concerned the impact of invasion of adjacent organs or structures or perforation or penetration through the peritoneum by carcinoma on overall survival. The issue was raised as early as 2000 at the College of American Pathologists Prognostic Factors Conference that penetration or perforation of visceral peritoneum by cancer should

The study was conducted on behalf of the Members of the AJCC Hindgut Task Force.

(C) Society of Surgical Oncology 2011

Published Online: 29 March 2011

J. M. Jessup, MD

e-mail: jessupj@mail.nih.gov have a worse prognosis than invasion of an adjacent organ or structure, especially if the cancer and all involved tissues were resected intact and with a negative margin. ${ }^{2}$ This was based on a careful pathologic study of resected colon carcinoma in which T4 lesions with peritoneal perforation/ penetration had a worse survival than when cancer invaded adjacent structures. ${ }^{3}$ Zeng and colleagues provided support for this in a single institution series where the incidence of free cells in the peritoneal cavity of T3 colon cancers was high and, thus, any involvement of the visceral peritoneum was expected to have a worse outcome. ${ }^{4}$ In contrast, another larger single institution study indicated that identification of carcinoma cells in the abdominal cavity did not portend a poorer prognosis in colon cancer, although it did in gastric cancer. ${ }^{5}$ As a result, the Hindgut Task Force felt that there was insufficient outcome data to recommend subsets for T4 for the 6th Edition of TNM for colorectal carcinoma but that data from larger data sets, preferably population-based would be performed for the 7th Edition of TNM and the Cancer Staging Manual. ${ }^{6}$ Such a review was performed on sets of patients with colon or rectal carcinoma not only from the Surveillance, Epidemiology and End Results (SEER) registry but also from cooperative oncology group trials. The analysis led to changes not only in the definitions of the subsets of $\mathrm{T} 4$ but also a reevaluation of the impact of both the number of nodes involved with cancer and the total number of nodes evaluated by the pathologist. In addition, the interplay between the depth of invasion of the bowel wall by carcinoma and the number of involved nodes was better delineated. ${ }^{7,8}$ 
Another goal for the Hindgut Task Force for the 7th Edition was to begin to consider how best to bring molecular markers into the staging system and to clinical practice. The analysis of the data sets suggests that there is interplay between invasion of bowel or other tissues and involvement of regional nodes that affects survival. Hopefully, this can be used to assess what molecular pathways are most significant for colon and rectal carcinoma outcome and lead ultimately to definition of molecular characteristics that are clinically useful. The Hindgut Task Force reviewed the literature and considered that the current data did not support the use of markers across stage groups and that anatomic pathology was still the most important prognosticators of outcome. However, there are several promising markers for which more data ought to be prospectively collected that may inform the 8th Edition of the TNM. These are included in the site-specific factors and the Hindgut Task Force urges their collection.

\section{HIGHLIGHTS OF THE 7TH EDITION OF THE AJCC COLORECTAL STAGING SYSTEM}

Key features of the 7th edition of the TNM staging system for colon and rectal carcinoma include the following:

- Expanded data sets indicated that differences in prognosis exist within T4 lesions based on extent of disease. Accordingly T4 lesions are subdivided as T4a (tumor penetrates visceral peritoneum) and as $\mathrm{T} 4 \mathrm{~b}$ (tumor directly invades or is histologically adherent to other organs or structures). The definitions of the T0-T3 lesions have not been affected since the 5th edition.

- The potential importance of satellite tumor deposits is now defined by the new site-specific factor tumor deposits (TD), which describe their texture and number. T1-2 lesions that lack regional lymph node metastasis but have tumor deposit(s) will be classified in addition as N1c.

- The number of nodes involved with metastasis influences prognosis within both $\mathrm{N} 1$ and $\mathrm{N} 2$ groups. Accordingly N1 will be subdivided as N1a (metastasis in 1 regional node) and $\mathrm{N} 1 \mathrm{~b}$ (metastasis in 2-3 nodes), and $\mathrm{N} 2$ will be subdivided as $\mathrm{N} 2 \mathrm{a}$ (metastasis in 4-6 nodes) and N2b (metastasis in 7 or more nodes). Both subsets represent $\sim 50 \%$ of patients in the $\mathrm{N} 1$ or $\mathrm{N} 2$ subset for colon or rectal cancer.

- Stage group II is subdivided into IIA (T3NO), IIB (T4aN0) and IIC (T4bN0) because the depth of invasion of the bowel wall in the absence of any positive regional nodes creates subsets with significantly different prognosis.

- Several changes were made in stage group III. A category of N1 lesions-T4bN1 - which was formerly classified as IIIB is reclassified IIIC largely because
T4b lesions have such a bad prognosis. In addition, several categories of N2 lesions formerly classified as IIIC have been reclassified IIIB because their prognosis is more favorable than previously considered (T1N2b, T2N2a-b, T3N2a, and T4aN2a). Similarly, T1N2a has been reclassified as IIIA. These changes were brought about because analysis of the large data sets indicated that the $\mathrm{T}$ category appears to be more closely associated with clinical outcome with thinner lesions having better outcome than thicker more penetrating lesions-even with similar numbers of pathologically involved lymph nodes (see reference Figs. 14-1 and 14-2; Chapter 14, pp. 149-1501).

- M1 has been subdivided into M1a for a single metastatic site vs. M1b for multiple metastatic sites. The Hindgut Task Force felt that this change should be made because clinical experience with resection and ablation suggests that outcomes are more favorable if metastases are limited to a single organ as opposed to having metastases in more than one organ. The Task Force felt that this is not a completely accepted finding but that by making this change the data can be prospectively accrued to assess whether it should be retained or altered in the next edition. There is no pM0 because it is not possible for a pathologist to ascertain the absence of distant metastases without an autopsy. The designation MX should generally be avoided, utilizing the $\mathrm{cM} 0$, $\mathrm{cM} 1$, or $\mathrm{pM} 1$ descriptors that may utilize imaging and/or pathological assessment.

- All of the new subdivisions of the T, N, and M categories and of the Stage groups can be collapsed into the main $\mathrm{T}, \mathrm{N}$, and $\mathrm{M}$ categories and stages of the previous TNM editions dating back to 1987. This ensures backwards compatibility not only to earlier TNM classifications but also to the Dukes classification.

\section{OTHER ISSUES FOR STAGING COLON AND RECTAL CARCINOMA}

The Hindgut Task Force feels that there are a number of unresolved questions that require prospective data collection to inform changes in the next edition of TNM. As information systems improve, these data will be easier to collect and to share to benefit public health. Specific areas of interest to the Hindgut Task Force include the following:

As with breast, pancreas, and perhaps other sites, there is an increasing use of neoadjuvant therapy. This applies primarily to rectal carcinoma where the trend during the past decade has been to give preoperative chemotherapy and radiation therapy to decrease the size of the primary cancer in an attempt to increase sphincter preservation and decrease the number of patients who need a permanent 
colostomy. A concern has been whether the response to neoadjuvant therapy is associated with overall survival. For that reason, one of the site-specific factors introduced in the 7th edition of TNM is the Tumor Regression Grade, which is based on the system of Ryan et al. except that grade is 0 for a complete pathologic response to neoadjuvant treatment and no viable tumor cells in the operative specimen, 1 for a moderate response with only single cells or small groups of cells present, 2 for a minimal response in which there is residual cancer with a desmoplastic response, and 3 for minimal evidence of tumor response. ${ }^{9}$ This applies generally only to rectal carcinoma and the initial classification will be based on a combination of clinical and pathological staging and the classification will be cTNM. Once the operative specimen is removed, the final postneoadjuvant response will be designated as per the standard TNM prefix of ypTNM. It is important that both the clinical pre-therapy classification and the post-neoadjuvant pathological classification be recorded so that for the 8th edition we have sufficient data to associate response to treatment with clinical outcome and evaluate the utility of the Regression Grading system.

Circumferential resection margin (CRM), especially for rectal carcinoma but also for those parts of the colon that are retroperitoneal, is another important issue. Popularized by Nagtegaal and Quirke, the resection margin is an important prognostic factor. ${ }^{10}$ The advent of total mesorectal excision (TME) by Cecil et al. dictates that a successful TME requires at least a 1-mm margin to be considered to have a negative resection margin. ${ }^{11}$ However, some data have indicated that a CRM should be closer to $1 \mathrm{~cm}$ to be considered negative because there is a possible association between the CRM in millimeters and local recurrence. Thus, the resection margin should be recorded on the pathology report and recorded as a sitespecific factor so that prospectively accrued data can be analyzed as to whether the margin should be wider to be considered negative. These prospective data should be used to determine whether any changes are needed for the next edition in terms of the recommendation for a negative margin.

An important related issue is the assessment of residual tumor (R), which is scaled on a R0 (all margins histologically negative after surgical resection), R1 (incomplete tumor resection with microscopically positive margins but without grossly evident cancer), and R2 (incomplete tumor resection with gross residual tumor) system. The $\mathrm{R}$ classifier is a long-term UICC/AJCC classification variable whose definition has not changed over the last several editions. However, there may be confusion between the CRM where the edge of the margin may be devoid of tumor cells in a high-powered field, but the CRM is positive because tumor cells are within $1 \mathrm{~mm}$ of the edge of the margin. In this instance, the Task Force felt that the $\mathrm{R}$ classifier and the CRM site-specific factor should be scored according to their current definitions even though it is now possible for a tumor to be R0 but $\mathrm{CRM}+$, essentially as described by Wittekind et al. ${ }^{12}$ By accruing data prospectively, it will be possible to determine whether the $\mathrm{R}$ classification needs to be modified possibly for rectal carcinoma.

There are several molecular factors that are close to acceptance for clinical use in the community if not already embraced by oncologists around the country. The most important is the presence of KRAS mutation as predictor of lack of response to antibody therapy to the epidermal growth factor receptor (EGFR). Clearly, current practice is for patients with advanced colorectal carcinoma who are considering treatment with an antibody to EGFR to be tested for the presence of activating mutations in KRAS. ${ }^{13}$ This can be performed on the original diagnostic fixed material and does not generally require a new biopsy because of high concordance of mutation status in primary tumors and metastases or recurrences. ${ }^{14}$ In addition, there are data that mutation in KRAS is associated with a worse prognosis than only wild type or "normal" KRAS. ${ }^{15}$ However, recent data also suggest that the importance of the presence of KRAS mutation may only apply to stage IV colorectal carcinoma and not apply to stage III or even II disease, because cetuximab has not shown benefit in the adjuvant setting in a recently reported clinical trial. ${ }^{16}$ Thus, the presence or absence of KRAS mutation is likely to be assessed only in a fraction of patients with advanced, unresectable systemic disease; only in those who may be treated with cetuximab or panitumumab.

Similarly, the other molecular markers that are being assessed in clinical trials are microsatellite instability (MSI) and loss of heterozygosity (LOH) of the short arm of chromosome 18 (18q). Data from institutional series and retrospective analysis of randomized clinical trials suggested that high levels of MSI (MSI-H) or $18 \mathrm{q}$ LOH may be important prognostic and perhaps predictive markers. The presence of MSI-H may suggest a better prognosis but predict a poor response to 5-fluorouracil (FU)-based chemotherapy, although the addition of irinotecan to 5-FU and leucovorin may be associated with an improved diseasefree survival in patients with stage III colon cancer. ${ }^{17-20}$ However, $18 \mathrm{qLOH}$ appears to be a marker for poor prognosis. ${ }^{18}$ An ongoing trial in stage II colon cancer (E5202 coordinated by the Eastern Cooperative Oncology Group) will determine prospectively the impact of $18 \mathrm{q} \mathrm{LOH}$ in patients who receive adjuvant chemotherapy with 5-FU in combination with oxaliplatin (FOLFOX), and the survival rate in patients with MSI-H tumors who are observed without adjuvant therapy. Should the current clinical trial demonstrate that MSI or $18 \mathrm{q}$ LOH is a useful marker for 
guiding therapy, it is critical that the status of the markers is available in large data sets through prospective ascertainment so that their impact in community practice can be assessed quickly. Furthermore, after the 7 th edition was completed, additional data accumulated that mutation of the BRAF gene in the RAS/RAF pathway is associated with poor survival in patients with colorectal cancer that lacks high levels of microsatellite instability (MSI-H) and may be a prognostic marker. ${ }^{21,22}$ As with KRAS mutation, it is not clear whether these molecular analyses will apply to multiple stages or be confined to limited clinical situations that are represented by single stage groups. Should the current clinical trial demonstrate that MSI or $18 \mathrm{q} \mathrm{LOH}$ is a useful marker for guiding therapy, it is critical that the marker values be collected in the TNM staging form so that their impact on community practice is assessed.

Finally, the issue of tumor deposits is an important anatomical finding that requires definition that may be assisted by collection of prospective data. Nagtegaal and Quirke have raised questions about enumeration of tumor deposits because of their potential association with clinical outcome. The Hindgut Task Force fully supported the concept of the biologic importance of tumor deposits and recommends the identification of tumor deposits by the definition in the staging manual: "Discrete foci of tumor found in the pericolic or perirectal fat or in adjacent mesentery (mesocolic fat) away from the leading edge of the tumor and showing no evidence of residual lymph node tissue but within the lymph drainage area of the primary carcinoma ${ }^{1}$, p. 151." There may be confusion as to whether a nodule of tumor in the mesentery adjacent to a cancer is replaced lymph node or a metastasis that is essentially not otherwise specified. Also it was not clear to the Hindgut Task Force whether these deposits were significant poor prognostic factors that could be recommended for inclusion in a staging system other than the instance where there is a tumor deposit in a patient with stage I or II disease lacking any positive nodes. In that circumstance, the Task Force recounted personal evidence that physicians across the country were beginning to treat these patients with adjuvant therapy as though they were stage III with a positive lymph node metastasis. The Task Force felt that adoption of the N1c classification was important if only to identify this situation and strongly recommends that the data about the prevalence of this situation as well as the clinical outcome be prospectively tracked.

\section{SUMMARY}

In the future, it should be possible to integrate increasingly molecular markers with TNM staging to measure prognosis and prediction of response to therapy. However, the markers in current use may not be the ones that will be useful for this purpose. The TNM staging system is based on anatomical pathology, is reproducible across many different clinical sites, and is the "gold standard" by which patients are diagnosed and treated around the world. Any subsequent use of molecular markers must be tailored to the pathological TNM and either enhance the core TNM stage groups or be able to represent it faithfully and as easily. Increasingly, gene expression profiles in multiplex qRT-PCR assays, such as Oncotype DX, which was developed initially for early stage breast cancer $^{23}$ and is now under development for colorectal cancer, ${ }^{24}$ or on chips, such as the Pathworks assay to assign an origin for tumors of otherwise unknown primary origin, ${ }^{25}$ are being developed, validated, and in process to be qualified for clinical use. Clearly, multiple groups have developed gene expression profiles that predict recurrence and death in and from colorectal carcinoma with varying degrees of accuracy and precision. ${ }^{26,27}$ Hopefully, by the time of the 8 th edition of TNM there will be an appropriate gene or protein expression profiles that will be a candidate that is close to clinical use. In the meantime, the 7th edition of TNM contains both a refinement of the interaction between tumor invasion and nodal metastasis as well as opportunities to garner prospective information that can further improve both the anatomic and molecular characteristics of the TNM stage grouping.

DISCLAIMER The views presented in this manuscript are those of the employees of the National Cancer Institute and do not necessarily represent those of the Federal Government.

\section{REFERENCES}

1. Edge SB, Byrd DR, Compton CC, Fritz AG, Greene FL, Trotti A. editors. AJCC cancer staging manual. 7th ed. New York: Springer; 2010.

2. Compton C, Fenoglio-Preiser CM, Pettigrew N, Fielding LP. American joint committee on cancer prognostic factors consensus conference: colorectal working group. Cancer. 2000;88:1739-57.

3. Shepherd N, Baxter K, Love S. The prognostic importance of peritoneal involvement in colonic cancer: a prospective evaluation. Gastroenterology. 1997;112:1096-102.

4. Zeng Z, Cohen AM, Hajdu S, et al. Serosal cytologic study to determine free mesothelial penetration of intraperitoneal colon cancer. Cancer. 1992;70:737-40.

5. Vogel P, Ruschoff J, Kummel S, et al. Prognostic value of microscopic peritoneal dissemination: comparison between colon and gastric cancer. Dis Colon Rectum. 2000;43:92-100.

6. Greene FL, Page DL, Fleming ID, et al. Colon and rectum. In: Greene FL, Page DL, Fleming ID, et al., editors. AJCC cancer staging manual. 6th ed. New York: Springer; 2002. p. 113-24.

7. Gunderson LL, Jessup JM, Sargent DJ, Greene FL, Stewart A. Revised tumor and node categorization for rectal cancer based on surveillance, epidemiology, and end results and rectal pooled analysis outcomes. J Clin Oncol. 2010;28:256-63.

8. Gunderson LL, Jessup JM, Sargent DJ, Greene FL, Stewart AK. Revised TN categorization for colon cancer based on national survival outcomes data. J Clin Oncol. 2010;28:264-71. 
9. Ryan R, Gibbons D, Hyland JMP, et al. Pathological response following long-course neoadjuvant chemoradiotherapy for locally advanced rectal cancer. Histopathology. 2005;47:141-6.

10. Nagtegaal ID, Quirke P. What is the role for the circumferential margin in the modern treatment of rectal cancer? J Clin Oncol. 2008;26:303-12.

11. Cecil TD, Sexton R, Moran BJ, Heald RJ. Total mesorectal excision results in low local recurrence rates in lymph nodepositive rectal cancer. Dis Colon Rectum. 2004;47:1145-9.

12. Wittekind C, Compton C, Quirke P, Nagtegaal I, Merkel S, Hermanek P, Sobin LH. A uniform residual tumor (R) classification: integration of the $\mathrm{R}$ classification and the circumferential margin status. Cancer. 2009;115:3483-8.

13. Allegra CJ, Jessup JM, Somerfield MR, et al. American Society of Clinical Oncology provisional clinical opinion: testing for KRAS gene mutations in patients with metastatic colorectal carcinoma to predict response to anti-epidermal growth factor receptor monoclonal antibody therapy. J Clin Oncol. 2009;27:2091-6.

14. Italiano A, Hostein I, Soubeyran I, et al. KRAS and BRAF mutational status in primary colorectal tumors and related metastatic sites: biological and clinical implications. Ann Surg Oncol. 2010;17:1429-34.

15. Russo A, Bazan V, Agnese V, et al. Prognostic and predictive factors in colorectal cancer: Kirsten Ras in CRC (RASCAL) and TP53CRC collaborative studies. Ann Oncol. 2005;16(Suppl 4): iv44-9.

16. Alberts SR, Sargent DJ, Smyrk TC, et al. Adjuvant mFOLFOX6 with or without cetuximab (Cmab) in KRAS wild-type (WT) patients (pts) with resected stage III colon cancer (CC): results from NCCTG Intergroup Phase III Trial N0147. Proc Am Soc Clin Oncol. (Abstract \#CRA3507). 2010 ASCO annual meeting.

17. Thibodeau SN, Bren G, Schaid D. Microsatellite instability in cancer of the proximal colon. Science. 1993;260:816-9.

18. Watanabe T, Wu TT, Catalano PJ, et al. Molecular predictors of survival after adjuvant chemotherapy for colon cancer. $N$ Engl $J$ Med. 2001;344:1196-206.
19. Sargent DJ, Marsoni S, Monges G, et al. Defective mismatch repair as a predictive marker for lack of efficacy of fluorouracilbased adjuvant therapy in colon cancer. $J$ Clin Oncol. 2010;28:3219-26.

20. Bertagnolli MB, Niedzwiecki D, Compton CC, et al. Microsatellite instability predicts improved response to adjuvant therapy with irinotecan, fluorouracil, and leucovorin in stage III colon cancer: cancer and leukemia. J Clin Oncol. 2009;27:1814-21.

21. Samowitz WS, Sweeney C, Herrick J, et al. Poor survival associated with the BRAF V600E mutation in microsatellite-stable colon cancers. Cancer Res. 2005;65:6063-9.

22. Roth AD, Tejpar S, Delorenzi M, et al. Prognostic role of KRAS and BRAF in stage II and III resected colon cancer: results of the translational study on the PETACC-3, EORTC 40993, SAKK 60-00 trial. J Clin Oncol. 2010:28:466-74.

23. Paik S, Tang G, Shak S, et al. Gene expression and benefit of chemotherapy in women with node-negative, estrogen receptorpositive breast cancer. J Clin Oncol. 2006;24:3726-34.

24. D. Kerr, R. Gray, P. Quirke, et al. A quantitative multigene RTPCR assay for prediction of recurrence in stage II colon cancer: selection of the genes in four large studies and results of the independent, prospectively designed QUASAR validation study [abstract 4000]. J Clin Oncol. 2009;27(Suppl):15s.

25. Wu AH, Drees JC, Wang H, et al. Gene expression profiles help identify the tissue of origin for metastatic brain cancers. Diagn Pathol. 2010;5:26.

26. Wang Y, Jatkoe T, Zhang Y, et al. Gene expression profiles and molecular markers to predict recurrence of Dukes' B colon cancer. J Clin Oncol. 2004;22:1564-71.

27. Smith JJ, Deane NG, Wu F, et al. Experimentally derived metastasis gene expression profile predicts recurrence and death in patients with colon cancer. Gastroenterology. 2010;138:958-68. 